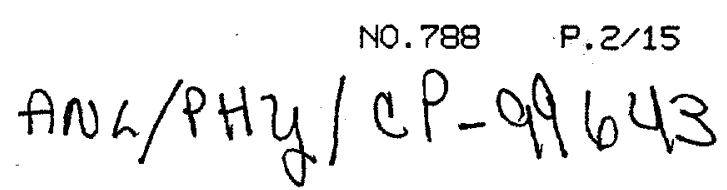

Submitted to the "International Symposium on Proton-Emitting Nuclei"

\title{
Fine Structure in Deformed Proton Emitters
}

\author{
A.A. Sonzogni, C.N. Davids, P.J. Woods ${ }^{1}$, D. Scweryniak, M.P. Carpenter, \\ J.J. Ressler ${ }^{2}$, J. Schwartz, J. Uusitalo, and W.B. Walters ${ }^{2}$. \\ Argonne National Laboratory, 9700 South Cass Ave, Argonne IL 60499
}

\begin{abstract}
In a recent experiment to study the proton radioactivity of the highly deformed ${ }^{131}$ Eu nucleus, two proton lines were detected. The higher energy one was assigned to the ground-state to ground-state decay, while the lower energy, to the ground-state to the $2^{+}$state decay. This constilutes the first observation of fine structure in proton radioactivity. With these four measured quantities, proton energies, half-life and branching ratio, it is possible to determine the Nilsson configuration of the ground state of the proton emitting nucleus as well as the $2^{+}$energy and nuclear deformation of the daughter nucleus. These results will be presented and discussed.
\end{abstract}

1 University of Edinburgh

${ }^{2}$ University of Maryland

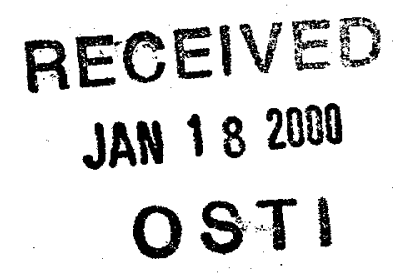




\section{DISCLAIMER}

This report was prepared as an account of work sponsored by an agency of the United States Government. Neither the United States Government nor any agency thereof, nor any of their employees, make any warranty, express or implied, or assumes any legal liability or responsibility for the accuracy, completeness, or usefulness of any information, apparatus, product, or process disclosed, or represents that its use would not infringe privately owned rights. Reference herein to any specific commercial product, process, or service by trade name, trademark, manufacturer, or otherwise does not necessarily constitute or imply its endorsement, recommendation, or favoring by the United States Government or any agency thereof. The views and opinions of authors expressed herein do not necessarily state or reflect those of the United States Government or any agency thereof. 


\section{DISCLAIMER}

Portions of this document may be illegible in electronic image products. Images are produced from the best available original document. 


\section{Introduction}

The proton drip-line meets a region of high quadrupole deformation around the light rare earth nuclei.[1]. Calculations by Möller ct al. [2] and Aboussir et al. [3] indicate deformations of $\sim 0.3-0.35$ for proton emitting nuclei ranging from La to Ho. There is sufficient experimental information confirming these ideas. In fig. I the energy of the first $2^{+}$state [1] is plotted as a function of neutron number for a number of different nuclei. Although the information for light rare earths is not particularly abundant, a trend is easily visible and for these nuclei, energies of around 100-150 keV can be expected. The discovery of highly deformed proton emitters [5] in this mass region prompted us to speculate about the existence of fine structure in proton radioactivity, i.e. the population of excited states in the daughter nuclei, since as it was just mentioned, the energy of the $2^{+}$state would be considerably low.

Because proton decay rates are very sensitive to the energy of the emitted proton, one may expect that chances of observing fine structure are quile small. For instance, if in the daughter nuclei there is a level at energy $E$, which we will assume can be populated with the same proton angular momentum as the ground state, the expression:

$$
B=T_{1 / 3}\left(Q_{g s}\right) /\left(T_{1 / 2}\left(Q_{g s}\right)+T_{1 / 2}\left(Q_{g s}-E\right)\right)
$$

can be used as a first approximation to estimate the branching ratio of this particular state, where $Q_{g s}$ is the ground-state proton Q-value. Using DWBA calculations - assuming spherical shapes - we conclude that if this hypothetical excited state lies at 100-150 keV, the estimated branching ratio appears to be smaller than $5 \%$ for rare earth nuclei with $Q_{g s} \sim .95 \mathrm{MeV}$. Deformed nuclei can effectively provide that low value of exeitation energy that it is needed, and despite the approximative nature of eq. 1, some insight can be obtained from it. If one aims at finding fine structure, one should produce the proton emitter with the highest deformation - and therefore lowest $\mathrm{E}_{2}{ }^{4}$ - and measure a proton energy spectrum with high statistics.

With these ideas in mind, an experiment was performed at Argonne $\mathrm{Na}$ tional Laboratory in August 1998. The results about this experiment have been already published and can be found in ref. [6], a few extra details and some ideas that have been developed since then will be discussed here.

\section{Experiment}

Nuclei of ${ }^{181} \mathrm{Eu}$ were produced through the reaction ${ }^{58} \mathrm{Ni}\left({ }^{78} \mathrm{Kr}, \mathrm{p} 4 \mathrm{n}\right)$ at $E_{l a b}=402$ $\mathrm{MeV}$. The Fragment Mass Analyzer [7] was used to separate the recoiling nuclei of interest. The detection system consisted of a PGAC - giving position and timing information - and a DSSD, where implant and decay events werc recorded. The DSSD was surrounded by extra Si detectors to veto out escape 
events. The calibration of the DSSD was performed with standard $\alpha$ sources and with protons from ${ }^{167 m} \mathrm{Ir}$. An energy spectrum of dceays events which oecurred within $100 \mathrm{~ms}$ of an implant event is shown in the upper panel of fig. 2. Two proton peaks can be seen, the higher energy one corresponds to the previously identified ${ }^{131} \mathrm{Eu}$ ground-state to ground-state proton transition ( $E_{p}=932(7) \mathrm{keV}, T_{1 / 2}=17.8(19) \mathrm{ms}$ ), and the lower one having $E_{p}=811(7)$ $\mathrm{keV}$ and $T_{1 / 2}=23_{6}^{10} \mathrm{~ms}$.

There are several scenarios that may explain these two peaks. One possibility is that they correspond to the decay of both an isomer and the ground state of ${ }^{131} \mathrm{Eu}$ into ${ }^{130} \mathrm{Sm}$, resembling what has been seen for nuclei like ${ }^{147} \mathrm{Tm}$ or ${ }^{167}$ Ir. For these spherical nuclei, isomerism arises from the nearly degenerate $2 \mathrm{~s} 1 / 2,1 \mathrm{~d} 3 / 2$ and $0 \mathrm{~h} 11 / 2$ orbitals and the fact that electromagnetic transitions from the $0 \mathrm{hll} / 2$ level to the other two, or vice-versa, will be highly hindered. The highly deformed surface shapes involved in ${ }^{191}$ Eu changes the situation completely eliminating this isomeriam. In fact, for deformed neutron-rich $\dot{\mathbf{P} m \text {, }}$ $\mathrm{Eu}, \mathrm{Tb}$ and Ho nuclei, no low-lying isomers were observed, due to the fact that the change in spin between different Nilsson orbitals is small enough so that gamma decay proceeds quite fast. In consequence, we are lead to think that we are observing the decay from the ground state of ${ }^{131} \mathrm{Eu}$ through two branches, which nicely explains the nearly identical value of experimcntal $T_{1 / 3}$. The higher energy line feeds the ground state of ${ }^{130} \mathrm{Sm}$ while the lower energy one, an excited atate of the same nucleus. With an excitation energy value of 121 $\mathrm{keV}$, the only option for this state is to be the $2^{+}$of the ground state rotational band. This energy agrees well with one may expect on the basis of previous measurements, as shown in fig. 1. Additionally, if the Grodzins' equation [9] is used to relate this value of $2^{+}$energy with $\beta_{2}$, we obtain a $\beta_{2}$ value of 0.33 , which agrees quite well with the predictions of Moller et al $\left(\beta_{2}=331\right)$ [2] and Aboussix et al $\left(\beta_{2}=.37\right)$ [3].

From the peals areas, the measured branching ratio to the $2^{+}$state is obtained as:

$$
B_{2^{+}}^{m}=\frac{\operatorname{Area}\left(2^{+}\right)}{\operatorname{Area}\left(2^{+}\right)+\operatorname{Area}\left(0^{+}\right)}
$$

and one may expect to deduce additional information about the decay process from the knowledge of this experimental quantity. However, before we can proceed, we need to remember that the decay of the $2^{+}$state in ${ }^{130} \mathrm{Sm}$ to the $0^{+}$can occur by two competing ways, an E2 photon or electron conversion. If a gamma ray is emitted, the probability that it will interact with the DSSD is negligible. However, if an electron is emitted, it may leave some of its kinetic energy in the DSSD, which will sum up with that left by the proton. For instance, if both electron and proton are fully stopped within the DSSD, the energy of the event will be equal to the energy of a ground-state to ground-state event. In consequence, the numbers of evenis in the lower energy peak is smaller that the total number of $2^{+}$decays, and $B_{2+}^{m}$, has to be considered a lower limit 
of the intringic value of branching ratio $B_{2+}$. To obtain the intrinsic value, a correction was applied to the measured one, which in this case was done through a MonteCarlo simulation of the DSSD. The input parameters of the simulation were the implantation depth of the ${ }^{131} \mathrm{Eu}$ nuclei, the energy of the $2^{+}$and the conversion coefficient of the $2^{+}$to $0^{+}$transition [8]. The lesson learned from this procedure is that a deep implantation will produce a large correction to $B_{2+}^{n}$, since the electrons are more likely to leave some energy in the DSSD. This is certainly a negative aspect of decp implantalion, which is counteracted by a large efficiency to detect protons, an important issue when one considers the very low production cross section for these nuclei. A shallow implantation, on the other hand, will minimize the correction factor, but decrease the efficiency of the DSSD.

As it is mentioned in ref. [6], most of these data was taken with a deep implantation, with the resulting branching ratio of $24 \pm 5 \%$. On the basis of what was discuseed in the Introduction, this value seems to be particularly high, understanding it reveals a new aspect proton emission from deformed nuclei.

\section{Formalism}

The proton decay width, from the initial state $J_{i} K_{i}$ feeding the ground state, is given by:

$$
\Gamma_{0+}=\Gamma_{J_{f}=0+j_{p} l_{p},}^{J_{1} K_{i}}
$$

where $j_{p}$ and $\ell_{p}$ are the total and orbital momentum quantum numbers of the emitted proton and

$$
\Gamma_{J_{j}=0+j_{y} l_{s}}^{J_{i} K_{i}}=2 \pi\left|B_{J_{j}=0+j_{s} l_{p}}^{J_{i} K_{i}}\right|^{z}
$$

with the transition amplitude $B_{J_{f}=0+j_{p} \ell_{y}}^{J_{i} K_{i}}$ given in refs. [5],[10]. For protons feeding the $2^{+}$atate,

$$
\Gamma_{2+}=\sum_{j_{\nu} \ell_{p}} \Gamma_{l_{s}=2+j_{p} \ell_{p}}^{J_{i} K_{i}}
$$

The total decay width is:

$$
\Gamma=\Gamma_{0+}+\Gamma_{2+}
$$

and the branching ratios are given by:

$$
\begin{aligned}
& B_{0+}=\frac{\Gamma_{0+}}{\Gamma_{0+}+\Gamma_{2^{+}}} . \\
& B_{2+}=\frac{\Gamma_{2+}}{\Gamma_{0+}+\Gamma_{2^{+}}} .
\end{aligned}
$$


For a proton in a prolate/oblate nucleus, the only good quantum numbers are the projection of its total angular momentum on the symmetry axis, $\Omega$, and its parity, $\pi$. The wave function for this proton can be written as:

$$
\Phi^{\Omega^{*}}=\sum_{\ell j} C_{\ell j}^{\Omega^{*}} \phi_{\ell j}^{\Omega^{*}},
$$

where $\ell$ and $j$ are the orbital and total proton angular momentum inside the nucleus and the expansion coefficients $C_{\ell_{j}}^{R^{*}}$ satisfy $\sum_{\ell j}\left|C_{\ell j}^{R^{*}}\right|^{2}=1$. For instance, a $3 / 2^{+}$wave function will look like:

$$
\Phi^{s / 3^{+}}=C_{2,3 / 2}^{3 / 2^{+}} \phi_{2,3 / 2}^{s / 2^{+}}+C_{2,3 / 2}^{3 / 2^{+}} \phi_{2,5 / 2}^{3 / 2^{+}}+C_{4,7 / 3}^{3 / 2^{+}} \phi_{4,7 / 2}^{s / 2^{+}}+C_{4,9 / 2}^{s / 2^{+}} \phi_{4,8 / 2}^{s / 2^{+}}+\ldots
$$

Due to angular momentum conservation, the decay to the $0^{+}$will require that $j_{p}$, be equal to $3 / 2$, but if it feeds the $2^{+}$state, $j_{p}$ can be equal $103 / 2,5 / 2$ and $7 / 2$. We will examine the contribution of each term to the $B_{2+}$ value. As was mentioned in the introduction, the term with $j_{p}=3 / 2$ will produce a percentual contribution in the single digit. The term with $j_{p}=7 / 2$ is of not particular significance, since its decay is further hindered by a large centrifugal barrier. The decay with $j_{p}=5 / 2$ on the other hand, has the potential of becoming important if $\left|C_{2,3 / 3}\right| \leq\left|C_{3,5 / 2}\right|$. In other words, the hindrance caused by the reduction in proton energy can be overcorne with a favorable proton wave function structure.

Similarly, a $5 / 2^{+}$wave function will look like:

$$
\Phi^{5 / 2^{+}}=C_{2,5 / 2}^{3 / 2^{+}} \phi_{2,5 / 2}^{s / 2^{+}}+C_{4,7 / 2}^{3 / 2^{+}} \phi_{4,7 / 2}^{3 / 2^{+}}+C_{4,9 / 2}^{3 / 2^{+}} \phi_{4,9 / 2}^{3 / 2^{+}}+\ldots
$$

A decay to the $2^{+}$will require $j_{p}=5 / 2,7 / 2$ and $9 / 2$. This time, however, the conditions that favor an enhancement in $B_{2+}$ are not present.

One then concludes that Niloson orbitals $\left[N \pi_{9} \Lambda\right] \Omega^{\pi}$, for which one can find an integer number $k$ so that $\Omega=N-2 k-1 / 2$, are likely to produce a higher $B_{2+}$ value than orbitals with $\Omega=N-2 k+1 / 2$. For instance the orbitals [411]3/2+ and $[532] 5 / 2^{-}$belong to the first group, while $[413] 5 / 2^{+}$and $[523] 7 / 2^{-}$belong to the second group.

When ${ }^{131} \mathrm{Eu}$ was first observed, it was not possible to diseern between the [411]1/2+ and [413]5/2 $/ 2^{+}$orbitals on the basis of proton energy and half-life alone. The results of our calculations are shown in fig. 3. Based on what was discussed on the previous paragraphs, it shouldn't come as a surprise that only the $[411] 1 / 2^{+}$orbital can produce a $B_{2+}$ value as large as the measured one.

\section{Energy dependance of branching ratio}

Fine structure with a measurable value of $B_{2}$ has been so far found in ${ }^{191} \mathrm{Eu}$. Other cases could be the lighter Eu isotopes, for which the proton Q-value is also larger. One may wondcr then what $B_{2+}$ values will be found for these 
nuclei. Fig. 4 shows the proton $T_{1 / 2}$ and $B_{2}+$ as a function of the proton energy for the [411]3/2+ orbital. A value of $E_{2+}$ of $120 \mathrm{keV}$ was assumed for these calculations. These results indicate a fast increase of the branching ratio with increasing proton energy. In particular, it may eventually reach the point where both ground state and $2^{+}$are populated with the same intensity.

The experimental observation of this feature will be very interesting. Unfortunately, production cross sections are quite small. Depending on the input parameters one uses in a Statistical Model calculation, we obtain values close to $1 \mathrm{nb}$ or smaller, which will requirc considerable amounts of beam time. In addition, the proton $Q$-values could be too large and a development of an experimental technique to study very fast proton emitters would have to be done.

This dependance of $B_{2+}$ with $Q$-value is also seen for $\alpha$ particles. Fig. 5 shows experimental values of $B_{2}$ for even-even, actipide alpha emitters for different values of $Q_{\alpha}$ [4]. The points are restricted to relatively constant values of $2^{+}$energies $(\sim 45 \mathrm{keV}$ ) to isolate the branching ratio dependance on $Q$-value. The increasing trend with increasing $Q_{\alpha}$ is easily seen, but with a more modest magnitude than for protons.

The cause of this difference between alpha and proton emitters can be found in the dependance of the respective decay rates with the $Q$-values, with protons being far more sensitive. This last statement can be more clearly visualized with the help of the WKB method [11] to calculate the penetrabilities:

$$
P=\exp (-2 G),
$$

where $G$ is the so-called Gamow factor. For even-even $\alpha$-emitters, $B_{2+}$ is basically given by:

$$
R_{\alpha}=P\left(\ell_{\alpha}=2, Q-E_{2+}\right) /\left(P\left(\ell_{\alpha}=0, Q\right)+P\left(\ell_{\alpha}=2, Q-E_{2+}\right)\right) .
$$

As we have seen, the expression for proton emitters is more complicated, but a rough estirnate can be obtained with:

$$
R_{p}=P\left(\ell_{p}, Q-E_{2+}\right) /\left(P\left(\ell_{p}, Q\right)+P\left(\ell_{p}, Q-E_{2+}\right)\right) .
$$

Plots of $R_{\alpha}$ (left panel) and $R_{p}$ (right panel) - where for simplicity we took $\ell_{\mathrm{p}}=2$ - as a function of $Q$ can be seen in fig. 6 . As can be appreciated, $R_{\alpha}$ changes little - from $17 \%$ to $30 \%$ - over a large range of $Q, R_{p}$ on the other hand, experiences a dramatic increase with increasing $Q$.

In summary, the results from the first observation of proton radioactivity fine structure have been presented and the interpretation discussed. Additionally, the dependance of fine structure probability with proton $Q$-value was investigated.

This work was supported by the U.S. Department of Energy, Nuclear Physics Division, under Contract W-31-109-ENG-38. 


\section{References}

[1] P.J. Woods and C.N. Davids, Annu. Rev. Nucl. Part. Sci. 47, 541 (1997).

[2] P. Moöller, J.R. Nix, W.D. Myers, W.J. Swiatecki, At. Data Nucl. Data Tables 59, 185 (1995).

[3] Y. Aboussir tt al., At. Data Nucl. Data Tables 61, 127 (1995).

[4] Table of Isotopes (8th edition), R. Firestone and V.S. Shirley, Editors. John Wiley and Sons (1996).

[5] C.N. Davids et al., Phys. Rev. Lett. 80, 1849 (1998).

[6] A.A. Sonzogni et al., Phys. Rev, Lett. 83, 1116 (1999).

[7] C.N. Davids et al, Nuel. Instrum. Meth. B70, 358 (1992).

[8] F. Rösel, H.M. Fries, K. Alder and H.C. Pauli, At. Data Nucl. Data Tables, 21, 91 (1978).

[9] L. Grodzins, Phys. lett. 2, 88 (1962).

[10] B.P. Bugrov and S.G. Kadmensky, Sov. J. Nucl. Plyys. 49, 967 (1989).

[11] S. Aberg, P.B. Semmes and W. Nazarewicz, Phys. Rev. C56, 1762 (1997); Phys. Rev. C58, 3011 (1998). 
Figure 1: Energy of the lowest $2^{+}$state as a function of neutron number for different nuclei.

Figure 2: Top panel: Measured proton energy spectrum from the decay of ${ }^{151} \mathrm{Eu}$. Bottom panel: Simulated proton energy spectrum.

Figure 3: Calculated $2^{+}$decay branching ratios for ${ }^{131} \mathrm{Eu}$ as a function of the quadrupole deformation parameter $\beta_{2}$, based on the $[411] 3 / 2^{+}$and $[413] 5 / 2^{+}$ Nilsson orbitals. The observed branching ratio lies in the band between the dashed lines.

Figure 4: Calculated $2^{+}$decay branching ratios as a function of the proton $Q$-value for the $[411] 3 / 2^{+},[413] 5 / 2^{+}$and $[523] 7 / 2-$ Nilsson orbitals respectively.

Figure 5: $B_{2+}$ for different even-even $\alpha$ emitters as a function of $Q_{\alpha}$.

Figure 6: Ratios of penetrabilities calculated using the WKB method for $\alpha$ emitters (left) and for proton emitters (right). 


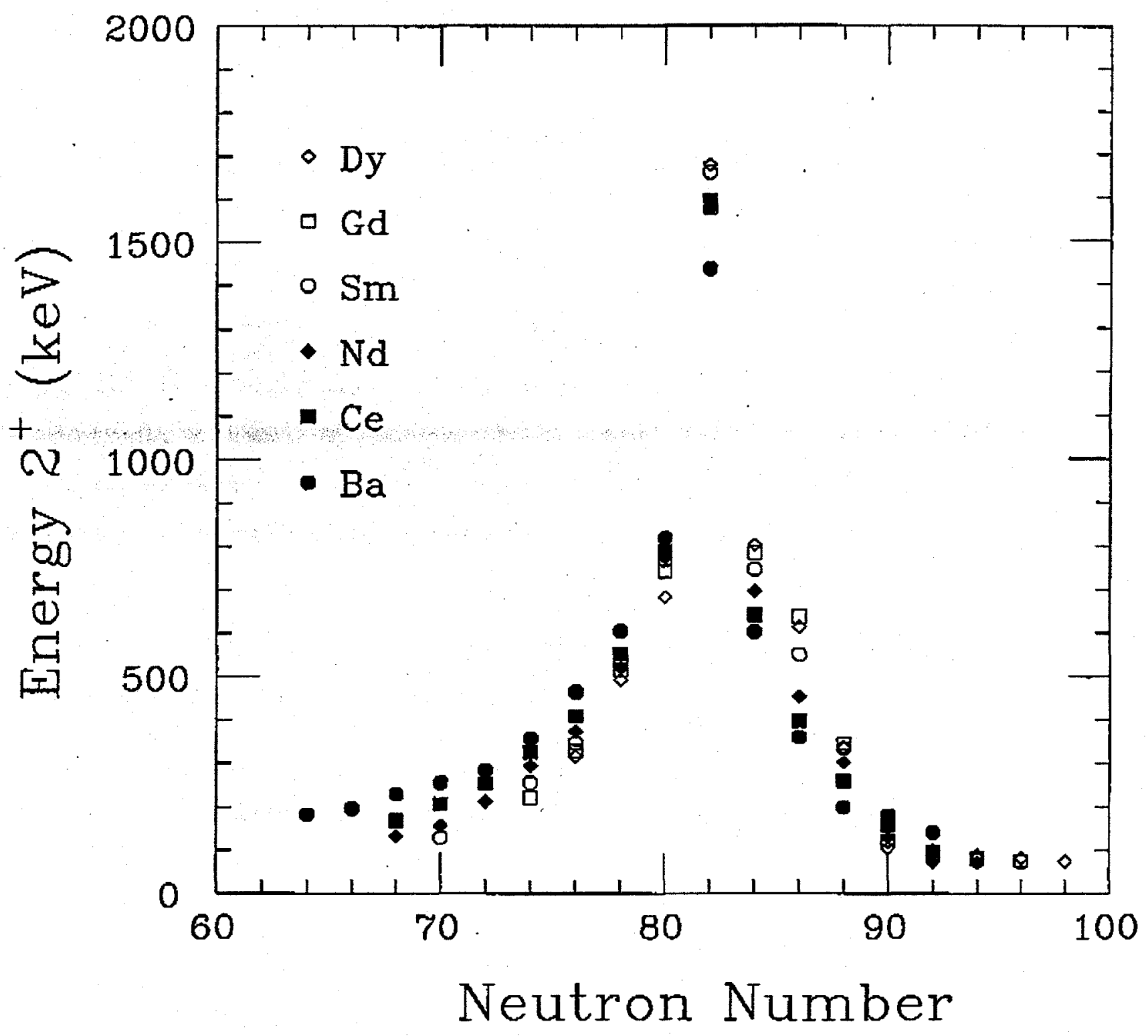




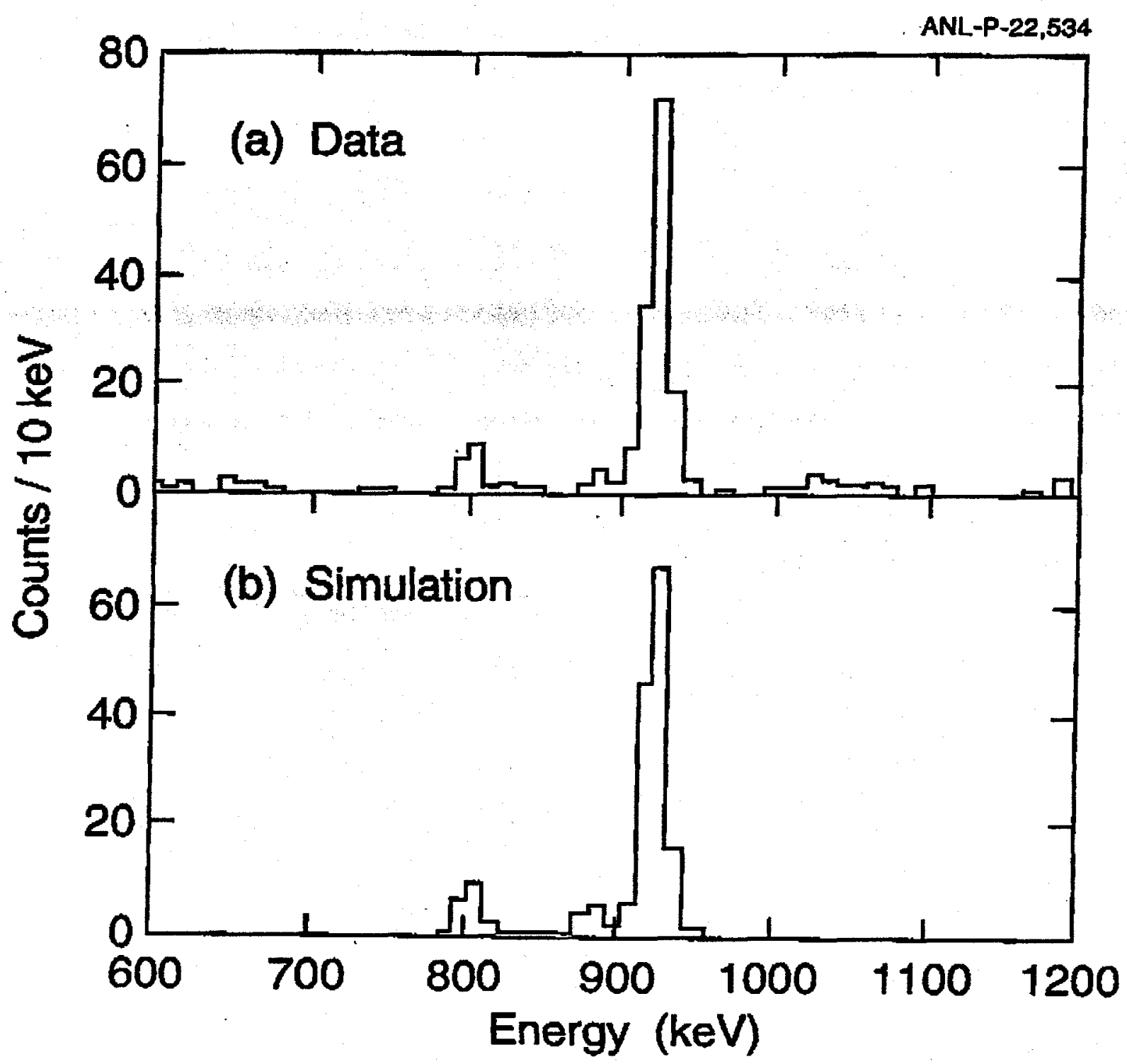


ANL-P-22.535

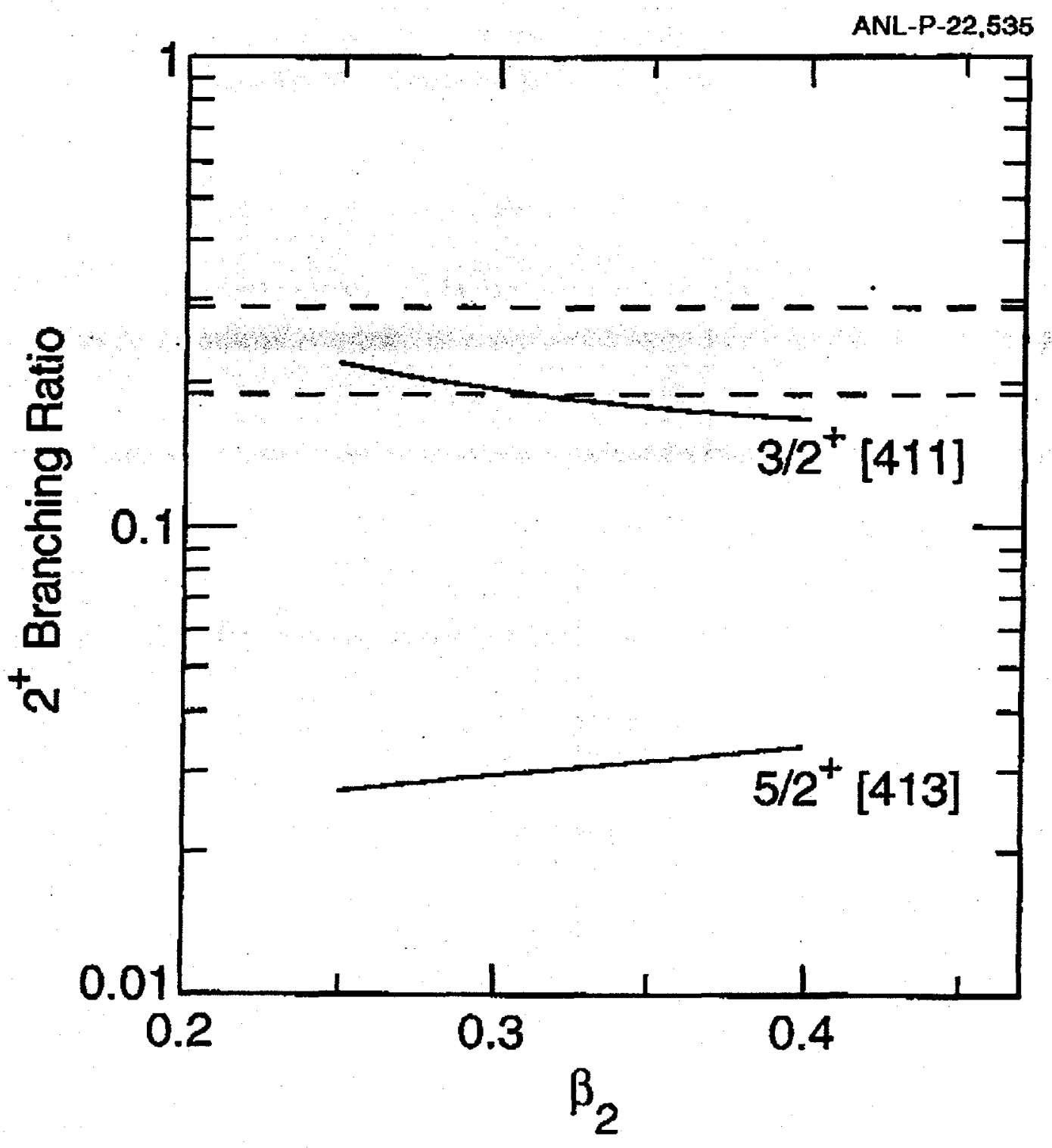



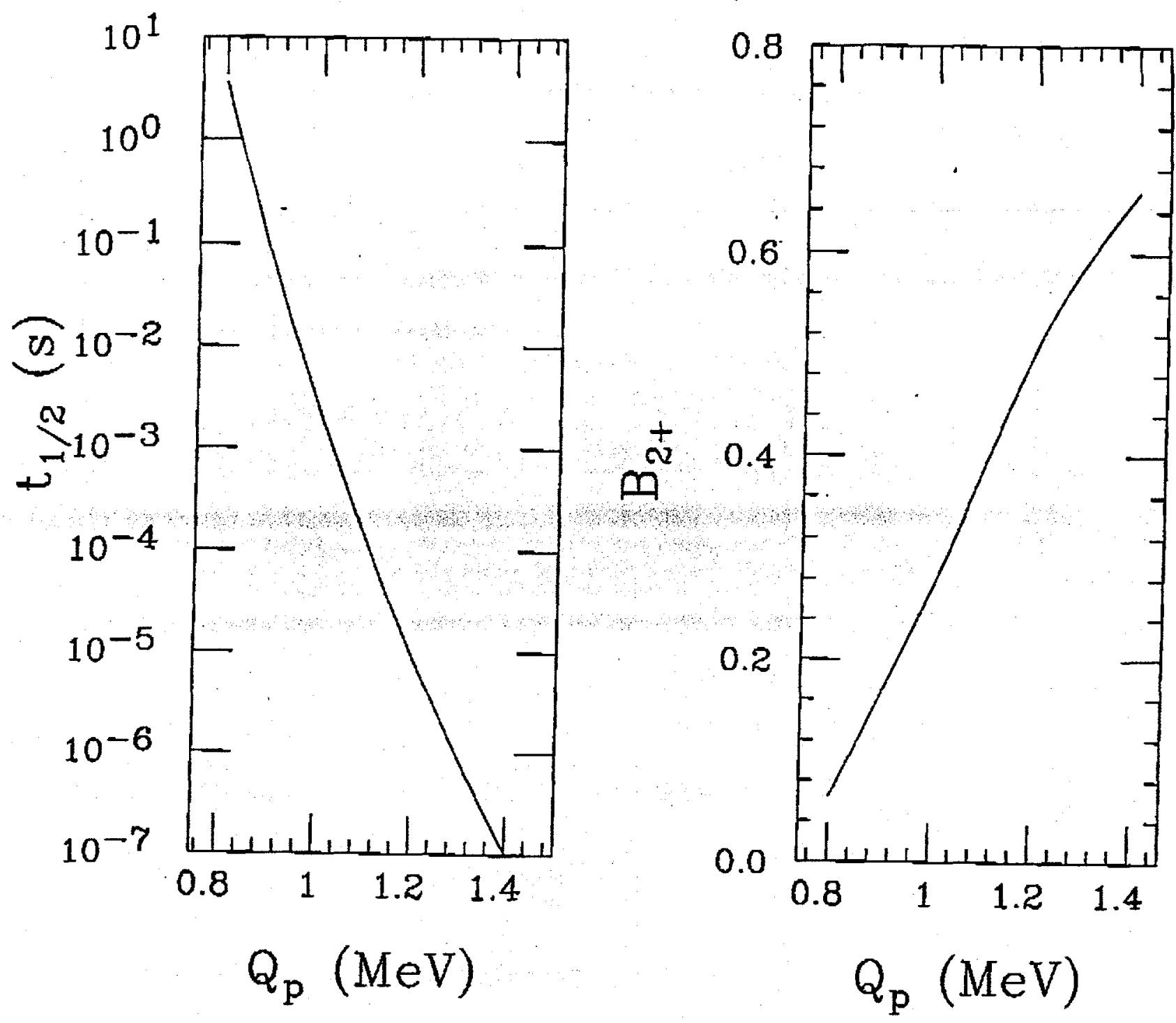
Tig. 5

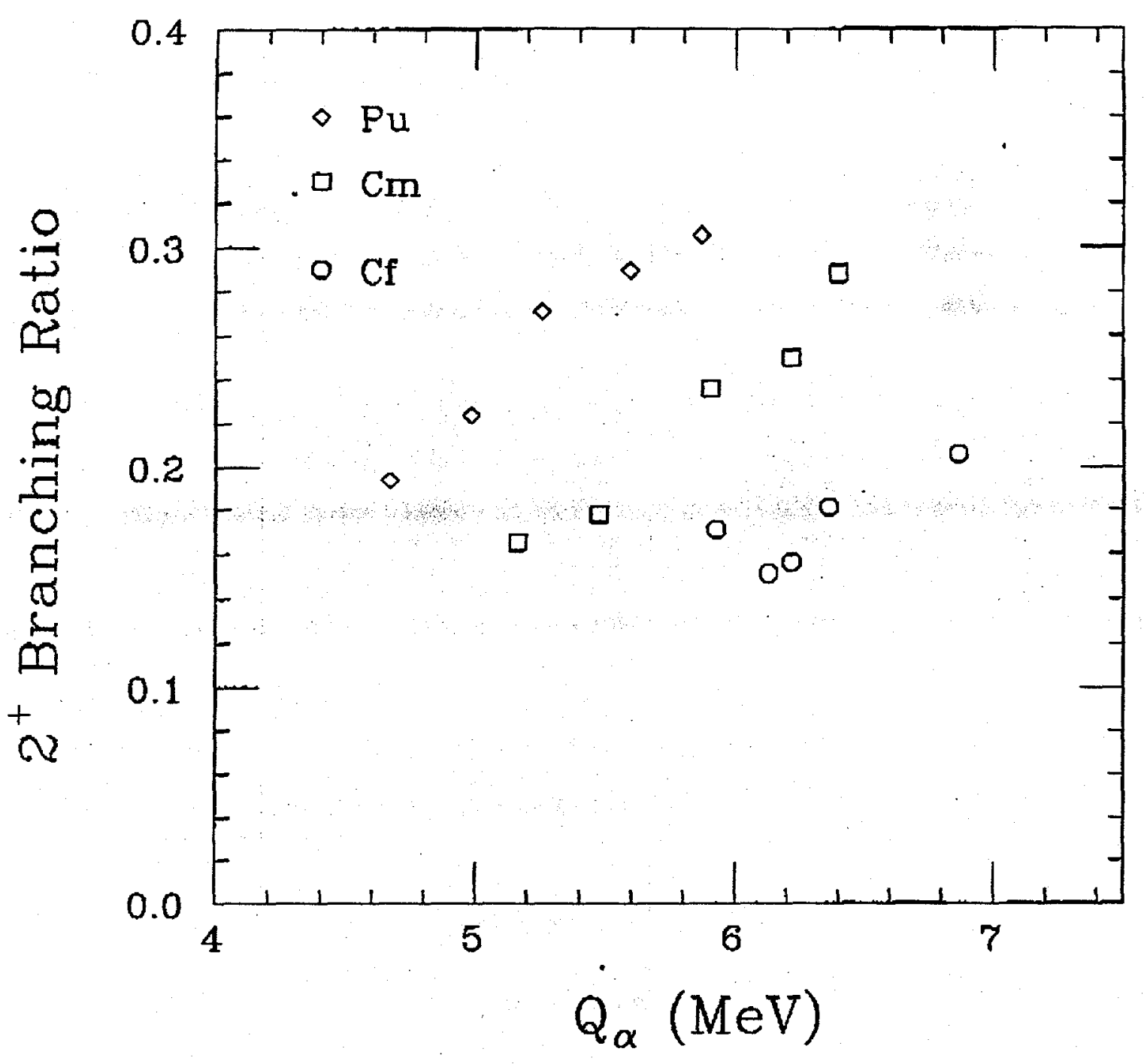


Tig. 6
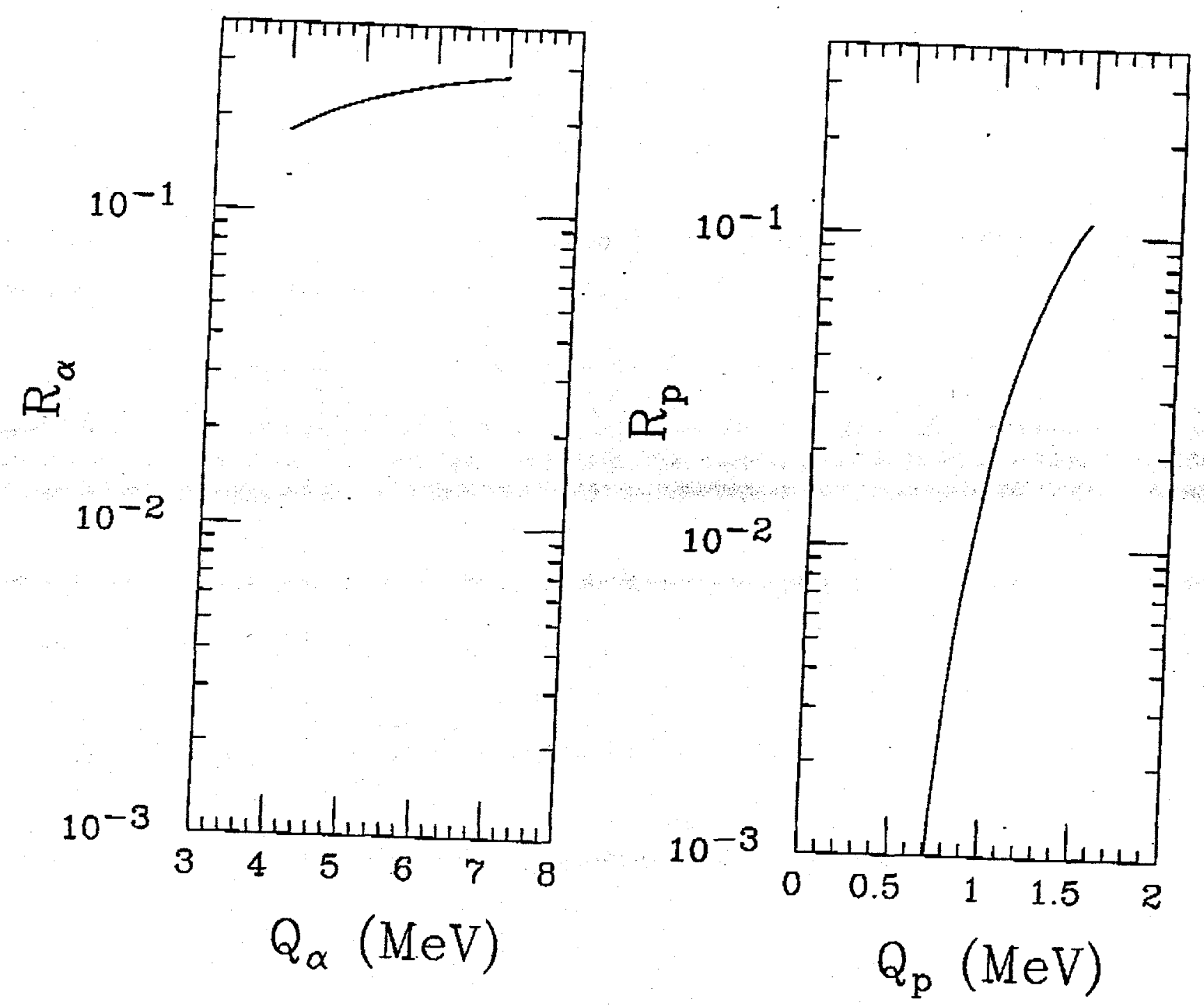